\title{
Mujer, embarazo y violencia \\ Síndrome de maltrato en la mujer embarazada
}

Alberto Moreno Rojas*

\section{RESUMEN:}

En el presente trabajo de investigación se muestra cómo la violencia, en las diferentes formas, que se ejerce en nuestro medio contra la mujer está produciendo una serie de síntomas confusos y desorientadores que no llegan a generar cuadros clínicos claros, que son de difícil manejo, por falta de conocimiento del medio ambiente en que vive nuestra paciente. -Toda esta sintomatología me ha llevado a proponer el "Síndrome de Maltrato en la mujer Embarazada"-.

MATERIALES Y METODOS. Se realizaron 150 encuestas entre pacientes, escogidas al azar, que fueron atendidas en la Clínica Federico Lleras A., del ISS de Neiva, y que presentaron problemas de comportamiento en el embarazo, el trabajo de parto, el parto y el puerperio.

RESULTADOS: Se han clasificado las pacientes de acuerdo con los hallazgos en tres grupos claramente definidos, con sus respectivas manifestaciones en cada uno de ellos, y se ha encontrado que existen uno o varios factores violentos desencadenantes.

CONCLUSIONES: La violencia que vivimos en nuestro país y que está produciendo manifestaciones en nuestras pacientes es un motivo más de preocupación y de estudio, para conocer mejor la situación, y poder ofrecer un apoyo Médico y Social a todo el grupo de mujeres embarazadas que necesitan la ayuda.

PALABRAS CLAVES: Mujer, embarazo, violencia.

\section{SUMMARY:}

In the present investigation work is show as the violence, in the different forms, that is exercised in our middle against the woman is producing a series of confusing symptons and bad orientation that they do not arrive to generate clear clinical tables, that they are of difficult managing, by lack of knowledge of the environment in which lives our patient. -All this symptomatology has carried me to propose the: "Mistreatment's in the pregnant Woman Syndrome"-.

MATERIAL AND METHODS: They were accomplished 150 polls among patient, chosen at random, that they were attended in the Federico Lleras A. clinic, of the ISS, Neiva, and that they presented behavior problems in the pregnancy, labor, delivery and the puerpery.

RESULTS: They has been classified the patients according to the findings in three groups clearly defined, with their respective manifestations in each of them, and they have been found that exist one or several violent factors unchains.

CONCLUSIONS: The violence that we lived in our country and that it is producing manifestations in our patient is a motive more than preocupation and of study, to know better the situation, and power to offer a social and medical support to all group of pregnant women that need the help.

KEY WORDS: Woman, pregnancy, violence.

\section{Introducción}

En la clínica Federico Lleras Acosta del ISS, Neiva, quienes allí laboramos en el área de Ginecobstetricia, hemos empezado a encontrar, cada vez con más frecuencia, una serie de síntomas y quejas por las cuales consultan nuestras pacientes y que son manifestaciones vagas, inconstantes y confusas que no dan lugar para establecer un cuadro clínico claro o poderlas encasillar dentro de una sintomatología de algún sistema específico, o que sean claras manifestaciones de un proceso crónico que se

\footnotetext{
* Ginecobstetra ISS. Neiva. Clínica Federico Lleras A.
}

agudizó con su estado gestacional, sino que corresponde, más bien, a un estado emocional debido a los múltiples problemas que le ocasiona su embarazo desde el punto de vista económico, social, habitacional, laboral, etc.

No quiere decir esto que todas las pacientes que consultan no presenten, en realidad, una sintomatología asociada o no a su embarazo, y, en algunos casos, cuadros clínicos y complicaciones de patologías de ocurrencia normal o de alguna frecuencia, los cuales reciben tratamiento adecuado con buenos resultados, pero sí hay un buen número de pacientes con aquella sintomatología, poco clara y confusa, en quienes todas las pruebas clínicas y exámenes de laboratorio son informadas como normales. 
Este es un grupo de difícil manejo, pues son consultantes crónicas del servicio de Urgencias, tanto generales como de obstetricia, y ocasionan acumulación de pacientes en la sala de espera, repetición de exámenes clínicos y de laboratorio y, en ocasiones, pasan a ser ocupantes de camas hospitalarias que se hacen necesarias para otras pacientes que, en realidad, si lo ameritan. De todas formas, son pacientes de mucho cuidado, pues, en ocasiones, toda esta sintomatología confusa está ocultando una verdadera patología, que puede resultar peligrosa, ya que estas pacientes le han dedicado más atención a los problemas y conflictos de su entorno que a la verdadera situación clínica que están padeciendo.

\section{Objetivo}

El objetivo de este trabajo clínico es poder mostrar, al menos en parte, cómo una serie de factores relacionados con el medio ambiente en que se desenvuelven nuestras pacientes, puede llegar a producir verdaderos cuadros clínicos que, con su sintomatología difusa, nos producen desorientación cuando llegan a nuestra consulta y como no conocemos el entorno en que vive y se desarrolla toda su problemática, por la cual nuestra paciente muestra esos estados de conducta anómalos, que la han llevado a generar toda una crisis con sus respectivas secuelas.

Se busca también, que, con los resultados encontrados y debidamente analizados, por las directivas de la institución, sirvan de base para tomar una serie de medidas y organizar a todas nuestras pacientes en verdaderos programas de acción social, los cuales, estando bien orientados, servirán como catalizadores a todo ese estado tensional que se presenta en nuestras mujeres embarazadas y que las lleva a ese rechazo hacia el resto de la sociedad, lo cual hace que se tornen agresivas y poco colaboradoras.

En realidad, este tipo de programas sociales no son de fácil ejecución por varias razones, que no son del caso examinar en este trabajo, pero si, al menos, después de dar el primer campanazo de alerta sobre una situación que, en cualquier momento, puede llevarnos a problemas mayores, se dan los primeros pasos en firme y se corrigen las fallas que se vayan presentando, de tal manera que, a la vuelta de unos pocos años, tendremos a todas nuestras pacientes embarazadas debidamente organizadas de acuerdo con sus necesidades y problemas, ofreciéndoles apoyo médico y social para ayudarles a encontrar una salida adecuada a sus conflictos, lo que se constituiría en un magnífico programa a desarrollar, en nuestra comunidad.

\section{Material y métodos}

Para realizar el presente estudio se tomó un grupo, escogido al azar, formado por 150 pacientes que habían presentado algunos problemas para su atención durante el embarazo, el trabajo de parto, el parto y el puerperio inmediato.

A todas las encuestadas se les realizó el interrogatorio de acuerdo con el protocolo establecido para su estudio y análisis posterior, con el fin de buscar una correlación entre las posibles causas de su sintomatología y la alteración de su conducta frente a los antecedentes familiares y personales de las pacientes.
Vale la pena anotar que no hubo rechazo, al interrogatorio, de parte de ninguna de las pacientes; por el contrario, se manifestaron francas y deseosas de diálogo, posiblemente, tratando de mostrarnos la necesidad que tenían de ser oídas y apoyadas.

Los principales motivos de consulta de nuestras pacientes que están sufriendo, de una u otra forma, del "Maltrato" durante su embarazo, se pueden enumerar así:

- Cansancio

- Sensación de aburrimiento

- Falta de sueño

- Fatigas

- Adormecimiento de los brazos y las piernas

- Sudadera

- Aburrida con el embarazo

- Tristeza permanente

- Deseos de dormir a toda hora

- Mucha sed

- Odio a los hijos varones y a mi esposo

- Orinadera

- Dificultad para la deposición

- Pocas ganas de trabajar

- Indisposición permanente

- Calambres en el estómago

- Pensamientos "raros"

- Vómitos permanentes

- Dolor de cabeza

- Dificultad para respirar

\section{Resultados}

Los resultados de las encuestas se detallan a continuación:

Tabla 1

GRUPOS DE EDAD

\begin{tabular}{|lll|}
\hline $15-19:$ & 20 & $(13.4) \%$ \\
$20-24:$ & 39 & $(26.0)$ \\
$25-29:$ & 49 & $(32.6)$ \\
$30-34:$ & 32 & $(21.4)$ \\
35 y $+:$ & 10 & $(6.6)$ \\
\hline
\end{tabular}

Tabla 2

GRADO DE ESCOLARIDAD

\begin{tabular}{|llll|}
\hline Primaria: & 51 & $(34.0) \%$ & \\
& Completa: & 30 & $(58.8)$ \\
& Incompleta: & 21 & $(41.2)$ \\
Secundaria: & 89 & $(59.4)$ & \\
& Completa: & 52 & $(58.5)$ \\
& Incompleta: & 37 & $(41.5)$ \\
Universitaria: & 9 & $(6.0)$ & \\
& Completa: & 5 & $(55.5)$ \\
& Incompleta: & 4 & $(45.5)$ \\
Analfabeta: & 1 & $(0.6)$ & \\
& & & \\
\hline
\end{tabular}


Tabla 3

TIPO DE VIVIENDA

\begin{tabular}{|llll|}
\hline Casa: & 119 & $(79.3) \%$ & \\
& Propia: & 58 & $(48.7)$ \\
Apartamento: & Arriendo: & 61 & $(51.3)$ \\
& 17 & $(11.3) \%$ & \\
& Propio: & 1 & \\
& Arriendo: & 16 & $(5.8)$ \\
& Inquilinato: & 14 & $(94.2)$ \\
& & & $(9.4) \%$ \\
\hline
\end{tabular}

Tabla 4

ESTADO CIVIL

\begin{tabular}{|lll|}
\hline Soltera: & 14 & $(9.4) \%$ \\
Casada: & 67 & $(44.6)$ \\
Separada: & 1 & $(0.6)$ \\
U. Libre & 68 & $(45.4)$ \\
\hline
\end{tabular}

Tabla 5

OCUPACION

\begin{tabular}{|lll|}
\hline Hogar: & 69 & $(46.0) \%$ \\
Empleada: & 75 & $(50.0)$ \\
Estudiante: & 6 & $(4.0)$ \\
\hline
\end{tabular}

Tabla 6

PARIDAD

\begin{tabular}{|lll|}
\hline 1: & 44 & $(29.3) \%$ \\
2: & 39 & $(26.0)$ \\
3: & 44 & $(29.3)$ \\
4: & 11 & $(7.4)$ \\
5: & 5 & $(3.3)$ \\
6: & 5 & $(3.3)$ \\
$7:$ & 2 & $(1.4)$ \\
\hline
\end{tabular}

Tabla 7

EDAD GESTACIONAL AL PARTO

\begin{tabular}{|lll|}
\hline De término: & 120 & $(80.0) \%$ \\
Pretérmino: & 30 & $(20.0)$ \\
\hline
\end{tabular}

Tabla 8

ESTE EMBARAZO FUE

\begin{tabular}{|c|c|c|}
\hline Deseado: & 64 & $(42.6) \%$ \\
\hline No deseado: & 86 & $(57.4)$ \\
\hline
\end{tabular}

Tabla 9

MOTIVO DEL EMBARAZO

\begin{tabular}{|lll|}
\hline Por tener un hijo: & 36 & $(24.0) \%$ \\
Por descuido: & 73 & $(48.6)$ \\
Por tener niño o niña: & 23 & $(15.4)$ \\
Por retener al esposo & 8 & $(5.4)$ \\
Por darle gusto al esposo: & 10 & $(6.6)$ \\
\hline
\end{tabular}

Tabla 10

SENTIMIENTO FRENTE A SU EMBARAZO

\begin{tabular}{|c|c|c|}
\hline Feliz: & 28 & (18.7) \% \\
\hline Satisfecha: & 29 & $(19,3)$ \\
\hline Triste: & 21 & $(14.0)$ \\
\hline No sabía que hacer: & 17 & (11.3) \\
\hline Preocupada: & 55 & $(36.7)$ \\
\hline
\end{tabular}

Tabla 11

DURANTE SU EMBARAZO, DESEO:

\begin{tabular}{|lll|}
\hline Abortar: & 20 & $(13.3) \%$ \\
Dar su hijo en adopción: & 10 & $(6.7)$ \\
Terminar pronto el embarazo: & 77 & $(51.3)$ \\
Buscar apoyo socioeconómico: & 43 & $(28.7)$ \\
\hline
\end{tabular}

Tabla 12

DURANTE SU EMBARAZO RECIBIO DE SU ESPOSO O COMPAÑERO:

\begin{tabular}{|lll|}
\hline Atenciones: & 47 & $(31.3) \%$ \\
Reclamos: & 36 & $(24.0)$ \\
Peleas ocasionales: & 26 & $(17.3)$ \\
Peleas permanentes: & 24 & $(16.0)$ \\
Agresiones verbales: & 11 & $(7.4)$ \\
Agresiones físicas: & 6 & $(4.0)$ \\
\hline
\end{tabular}

Tabla 13

EN SU TRABAJO RECIBIO

\begin{tabular}{|lll|}
\hline Apoyo: & 20 & $(26.7)$ \\
Advertencias: & 25 & $(33.3)$ \\
Reprimendas: & 9 & $(12.0)$ \\
Amenazas de despido: & 17 & $(22.7)$ \\
Cambio de cargo: & 4 & $(5.3)$ \\
\hline
\end{tabular}

Tabla 14

ACTITUD DE SU FAMILIA

\begin{tabular}{|lll|}
\hline Apoyo: & 67 & $(44.7) \%$ \\
Rechazo a su estado: & 31 & $(20.7)$ \\
Recriminaciones: & 26 & $(17.4)$ \\
Retiro del apoyo: & 18 & $(12.0)$ \\
Sugerencias de abortar: & 8 & $(5.2)$ \\
\hline
\end{tabular}

Tabla 15

ACTITUD FRENTE A SU HIJO

\begin{tabular}{|lll|}
\hline Contenta: & 56 & $(37.3) \%$ \\
Preocupada por su futuro: & 58 & $(38.7)$ \\
Preocupada factor económico: & 27 & $(18.0)$ \\
Preocupada rechazo familiar: & 9 & $(6.0)$ \\
\hline
\end{tabular}


En los casos clínicos revisados, seguidos y atendidos en la institución, se ha encontrado, lo siguiente:

Un primer grupo, formado por aproximadamente el $25 \%$, son pacientes con dificultades en su relación Médico-paciente, mostrándose rebeldes y negativistas, lo que dificulta la práctica del examen de ingreso y los controles durante el trabajo de parto y parto, por su agresividad, y, desde luego, falta de colaboración con el personal médico y paramédico.

No es extraño encontrar pacientes, en este grupo, que vociferan y lanzan improperios contra su esposo, familiares y todo el personal que pretenda ofrecerle alguna ayuda.

La atención del parto, en este grupo, está caracterizada por la dificultad para lograr que esa paciente facilite su atención en la sala de partos, llevando en ocasiones, su agresividad, a no tolerar que se le atienda en la forma adecuada en la mesa obstétrica y se termine atendiendo el parto en el piso de la sala de partos o en una de las camas de la sala de trabajo de parto, con todos los problemas obstétricos que esto puede ocasionar. Cuando se programa para cirugía (Cesárea), indicada en muchas ocasiones por la dificultad para su control, el problema es para el anestesiólogo y el personal de salas de cirugía, pues su colaboración es mínima, para poder realizar un manejo adecuado a su estado gestacional.

Un segundo grupo, formado aproximadamente por el $60 \%$, son pacientes que ingresan en un estado de menor agresividad contra médicos y personal paramédico, que el grupo anterior, pero a medida que empiezan a ver y sentir que lo que se pretende es ayudarlas a llegar a una feliz culminación de su embarazo, la mayoría, modifican notoriamente su conducta y se hacen receptivas a las indicaciones médicas, logrando, de esta manera, un buen resultado tanto por vías naturales como quirúrgicas.

$Y$ un tercer grupo, formado por el $15 \%$ restante, que llegan a la institución en un estado de desinterés marcado, donde la única preocupación que manifiestan es "salir pronto de su problema", sin importar la vía ni el resultado. La colaboración de la paciente es, desde luego, muy pobre y la tendencia a las crisis depresivas, leves y moderadas, en el puerperio inmediato, tratadas con ayuda del psicólogo, son muy frecuentes.

Los estados de Sicosis Puerperal se han manifestado en 2 de las pacientes del presente estudio y han seguido, en control, con el Psiquiatra.

Durante el tiempo que duró el presente estudio se atendieron 30 partos prematuros, en las pacientes encuestadas, y no se presentaron óbitos fetales que tuvieran relación alguna con la alteración de la conducta presentada durante el trabajo de parto y el parto.

Con toda esta sintomatología, hallada en nuestras pacientes, todo el cuadro sicoemocional que ocasiona y las formas de manifestación que presenta, he pretendido de una manera sencilla pero buscando colaborar para poder entender mejor a estas pacientes, darle el nombre de "Síndrome de Maltrato en la mujer embarazada". La principal razón de incluir dentro de un síndrome toda esa sintomatología confusa y las alteraciones de conducta, considero que, es la mejor manera de tratar de conocer un problema con el que cada día nos estamos encontrando y, de esta forma, tener los fundamentos necesarios para poder organizar un sistema de apoyo y orientación para la comprensión y el manejo del cuadro clínico.

\section{Comentarios}

Revisando la amplia bibliografía referente al tema de la violencia familiar y en pareja, he extractado algunos comentarios de autores que muestran un claro panorama del posible origen y de la evolución del problema en nuestro medio. En su libro: "La pareja. Cómo vivir juntos", de N. Rojas de González, dice: "A fin de que podamos comprender la dinámica de la violencia en la pareja colombiana actual, es necesario revisar las diferencias en los roles sexuales del hombre y de la mujer, impuestos y vigentes desde la época de la conquista, y que en cada región tiene sus manifestaciones propias. En todo el país se destaca un denominador común que es el poder de la organización patriarcal en detrimento de la posición de la mujer y de los hijos como miembros de la familia y de la sociedad. Dicho sistema es apoyado por el Estado y por la Iglesia para mantener al "Jefe del Hogar" con la plenitud de sus derechos. Esto es reforzado, además, por la forma de distribución de la autoridad existente en Colombia que, con sus implicaciones económicas, no sólo ha detenido el cambio social sino también el desarrollo pleno de las comunidades y de las clases proletarias".

"Los hombres que golpean a sus mujeres tienen un mal concepto de si mismos y, por lo tanto, reaccionan con hostilidad cuando su masculinidad y su autoridad se ponen en duda. A su vez, las mujeres que son objeto del maltrato físico presentan una baja autoestima y tienen dificultad para hacer valer sus derechos".

"El temor es un común denominador de las mujeres que se dejan golpear. En las circunstancias en que se han visto sujetas a repetidos abusos, el miedo las paraliza y rige sus actos, sus decisiones y sus vidas. No tratan de cambiar la situación mediante la denuncia o búsqueda de apoyo; piensan que no volverá a suceder".

Ma. Himelda Ramírez en su libro: "Mujer, Amor y Violencia", comenta: "Una clara demostración de la certeza que tiene el padre o sustituto, de la magnitud de su poder, se expresa en las reiteradas amenazas explícitas o implícitas que éste profiere, de abandono del hogar, cuando se presentan elementos de fricción o de conflicto, o cuando hay intentos de plantearle algunas exigencias. En muchos hogares tales amenazas no son infrecuentes, y son formas de hostilidad muy lesivas. Suelen ser, sin embargo, bastante afectivas para lograr imposición de determinados criterios y para la conservación de los privilegios de que disfruta el padre".

En su enfoque sobre la violencia doméstica, Melba Arias de Londoño en su libro: "Cinco formas de violencia contra la mujer", escribe: "Esta modalidad se convierte en el arma más eficaz para someter y doblegar voluntades y perpetuar costumbres sagradas dentro del hogar, especialmente las atinentes a roles, pues mientras más necesidad tenga el varón de reforzar su posición de jefe, más agresividad desarrolla contra los "subalternos". Y mien- 
tras más dependencia económica y afectiva tenga la mujer, es más susceptible de ser agredida. Por ello es que la violencia doméstica, como pan de cada día en muchos hogares, constituye un verdadero atentado contra la familia y la sociedad".

"Entre los factores desencadenantes de este maltrato familiar, lógicamente se encuentran las dificultades socioeconómicas (Desempleo, alcoholismo) y los problemas sicológicos (Stress, inseguridad, desconfianza, desamor), situaciones todas que producen en sus miembros diferentes sentimientos de hostilidad, ira, angustia, resentimiento, como manera de enfrentar los fracasos".

Complementa el tema, Ana Rico de Alonso, en su libro: "Madres Solteras Adolescentes", cuando dice: "A nivel familiar, la primera reacción de padres, hermanos y parientes siempre es negativa. En casos extremos, la joven es arrojada del hogar; en la mayoría, es agredida verbal y/o físicamente, recluida y encerrada para evitar la vergüenza y la deshonra. De todas maneras, las relaciones afectivas intrafamiliares se afectan, en muchos casos, de forma irreversible. El enterarse del embarazo de la hija, generó reacciones violentas y negativas: insultos, golpes, mal primero y luego la apoyan, furia, la echaron de la casa, indiferente, supieron después del parto, estupor, vergüenza, otras (presión, aborto y reclusión)".

En algunas regiones del Caribe, según relata Eduardo Galeno, en su libro: "Las venas abiertas de América Latina", los mayorales descargaban sus látigos de cuero o cáñamo sobre la espalda de las esclavas embarazadas que habían incurrido en falta, pero no sin antes acostarlas boca abajo, con el vientre en un hoyo, para no estropear la "pieza" nueva en gestación".

\section{Conclusiones}

El momento actual que vive nuestra sociedad, finales del siglo XX y albores del siglo XXI, donde la insensibilidad social y la falta de solidaridad de nuestras gentes ha entrado a formar parte de su vida diaria; donde la violencia ha ingresado por la puerta principal de muchos hogares llevándolos, prácticamente, a su destrucción; donde a la mujer y a los hijos les toca vivir los resultados de ese caos social que está acabando con ese núcleo tan importante como es la FAMILIA; donde los diferentes gobernantes que han dirigido nuestro país nos muestran resultados positivos de todas las promesas de cambio social que hacen en sus campañas; donde a quienes nombran para dirigir esos "Programas Gubernamentales" son los menos idóneos, y, desde luego, incapaces para desarrollar un programa social si es que, en realidad, tal programa y voluntad de ayuda existe; entonces, vale la pena hacer un alto en el camino y ponernos a pensar como médicos y como parte integrante de esa "Sociedad enferma", donde no solamente nuestras pacientes están sufriendo de sus propias enfermedades sino, también, las producidas por su ambiente violento, y donde la mujer se ha constituido como el objetivo y receptora de toda esa violencia, la cual se manifiesta de una forma franca o callada, sea ya en el hogar, en la calle, en el trabajo o en cualquier actividad donde, ella, esté comprometida. De acuerdo con esta idea, es nuestro propósito dedicarnos a conocer más de cerca esta dolorosa y difícil situación de nuestras pacientes, trazarnos unas metas y proponer unos programas donde podamos orientar, proteger y prevenir, al menos en parte, los daños que se pueden causar y que se lograrían evitar si practicamos una medicina donde conozcamos mejor el entorno en que se desenvuelve nuestra paciente e incluirla, a las que más lo necesiten, en programas de asistencia social a sus necesidades.

Después de conocer los resultados de las encuestas realizadas, relacionarlos con los síntomas y alteraciones de conducta, encontradas en las pacientes del presente estudio, y de reunir todas esas manifestaciones, dentro de un síndrome, es una buena forma para comprender que los diferentes factores desencadenantes de la violencia que afectan a la familia y a la mujer, en especial, deben ser un motivo de interés y de estudio, para conocer más de cerca esa dura realidad que nos está mostrando el evidente problema médico y social, que vivimos en la actualidad, y en el cual tenemos, como profesionales de la salud, unos programas por desarrollar y unas metas que cumplir.

\section{BIBLIOGRAFIA}

1. Rojas de González Nelly. La pareja. Cómo vivir juntos. $3^{\text {a }}$ Edición. Bogotá. Editorial Planeta. 1995; 182.

2. Ramírez Ma. Himelda. Mujer, Amor y Violencia. $2^{a}$ Edición. Bogotá. Editorial Tercer Mundo. 1991; 113.

3. Arias de Londoño Melba. Cinco formas de violencia contra la mujer. $1^{a}$ Edición. Bogotá. Editorial Colombia Nueva Ltda. 1990; 54.
4. Rico de Alonso Ana. Madres solteras adolescentes. $1^{a}$ Edición. Bogotá. Editorial Plaza y Janes. 1986; 40 y 86.

5. Galeano Eduardo. Las venas abiertas de América Latina. $60^{\mathrm{a}}$ Edición. Bogotá. Editorial Siglo XXI. Editores de Colombia. 1990; 135. 